\title{
Transitioning into obesity
}

\section{Pandey AS}

Arti S. Pandey, Professor; Department of Biochemistry, Kathmandu Medical College, Public Limited, Kathmandu, Nepal.

DOI: https://doi.org/10.3126/jkmc.v8i1.25386

$\mathrm{O}_{\mathrm{Bach}}^{\mathrm{b}}$ besity is defined as a physical state of having a Body Mass Index (BMI) greater than $30 \mathrm{Kg} / \mathrm{m}^{2}$. It is associated with an increased risk of many metabolic disorders like type 2 diabetes mellitus (T2DM) as well as with cardiovascular diseases (CVD), musculoskeletal disease, Alzheimer's disease and some types of cancers. In simple terms, obesity is a result of an imbalance between energy input and energy expenditure. As human beings evolved, natural selection was diverted more towards storage of food when it was available only after demanding physical efforts of hunting or working in the fields to grow food. As manual labor was gradually replaced by machines, physical activity of humans was drastically reduced. Instead, an office culture initiated a sedentary lifestyle, requiring long hours of sitting at desks. Parallel to this change in society, trade prospered amongst countries all across the globe, assuring the constant availability of food to all. The consequences of over-nutrition had not been considered, and gradually the mortality rates due to over-nutrition have become just as concerning as those due to under-nutrition.

In theory, obesity can be reversed if a person spends more energy and reduces energy input in the form of calories. In practice, however, interventions aimed at this reversal have not been effective. This is because many complex regulatory factors including hormones and neurotransmitters determine whether energy will be stored in the form of triglycerides in the adipose, or used up to generate energy in the form of adenosine triphosphate (ATP) to fuel various bodily mechanisms. Food intake is a response to stimulation of centers in the hypothalamus which send out orexigenic signals, stimulate gastrointestinal activity and receive satiety signals. Both short term and long term signals are involved in orchestrating ingestive behaviors, with gastrointestinal hormones largely regulating food

Address for correspondence

Dr. Arti S. Pandey

Professor, Department of Biochemistry

Kathmandu Medical College, Duwakot, Bhaktapur

E-mail: arti.pandey69@gmail.com intake acutely, while insulin and leptin provide long term regulation'. Gut peptides like cholecystokinin, pancreatic polypeptide, peptide YY, glucagon-like peptide-1 and oxyntomodulin increase satiety, while another gut peptide, ghrelin is a signal for hunger ${ }^{2}$. Their sites of action are located in the central nervous system, especially the arcuate nucleus of the hypothalamus. Melanocortin, neuropeptide $Y$, serotonin, dopamine and noradrenaline functions in the hypothalamus maintain energy balance via both the intake and expenditure pathways. Food intake hence, is a highly complex process that is regulated by many factors and not just a desire to eat.

The body undergoes major changes in its metabolic pathways as it transitions into obesity. Obesity is associated with increased triglyceride storage and reduced lipid mobilization from these stores. The adipocytes in an obese individual are both larger as well as higher in number. The adipose tissue produces many protein signals including growth factors, cytokines, chemokines, acute phase proteins and adhesion molecules. One of these is leptin, which acts as a satiety signal at the hypothalamus to limit food intake. The hypothalamus neurons become leptin resistant in obesity. Another adipokine, adiponectin, which is essential for tissues to respond to insulin, is decreased in obesity while resistin, which induces insulin resistance, is increased ${ }^{3}$. The cytokine Tumor Necrosis Factor (TNF) and other inflammatory cytokines are secreted by the adipose tissue macrophages (ATMs), which accumulate in large amounts in the adipose during obesity ${ }^{4}$. These inflammatory cytokines contribute to insulin resistance of tissues by interfering with the mechanism of action of insulin. Hence, through insulin resistance, obesity causes a lack of glucose uptake into the skeletal muscle and adipose tissues, resulting in hyperglycemia, which later develops into T2DM. There is an increase in mobilization of fat stores from the adipose due to insulin resistance as well as due to the adipokine SAA (serum amyloid A). There is a consequent rise in free fatty acids in the blood and accelerated rate of synthesis of very low density lipoproteins (VLDLs). SAA also interferes with the anti- 
atherogenic activity of High density lipoproteins (HDL). The resulting dyslipidemia increases the risk of coronary artery disease.

A predominantly upper body fat distribution, commonly associated with increased visceral fat, increases the risk of dyslipidemia, hypertension and T2DM. Lower body fat on the other hand, is associated with a reduced risk of these pathologies. Central adiposity is a result of accumulation of fat around the organs in the abdominal cavity of which the intraperitoneal fat a.k.a the visceral adipose tissue (VAT), is associated with insulin resistance, glucose intolerance, dyslipidemia and cardiovascular disease. Subcutaneous fat and circulating lipoproteins contribute to accumulation of triglycerides in the liver causing Non alcoholic fatty liver disease (NAFLD) which can lead to dyslipidemia and coronary heart disease.

The Nepal Non-communicable Diseases (NCD) Risk factor survey ${ }^{5}$, based on BMI measurements, estimated a prevalence of obesity of $4 \%$ in Nepalese men and women, while $17.7 \%$ were found to be overweight (BMI $=25-29$ ). The study also found a quarter of the Nepalese population aged 15-69years to have high blood pressure. This number is likely to be higher if restricted to regions of Nepal that have undergone rapid urbanization. The urban regions of Nepal have witnessed a radical cultural change due to the development of processed food industry within the country as well as flooding of markets with food products that have been imported from neighboring countries. Coupled with economic growth and an improvement in living standards, there has been an increasing trend for previously unexplored food. Traditional food in Nepal includes rice, maize, millet, leafy vegetables, lentils and dairy products. Snacks have traditionally been bean based and fermented foods were home made with leaves and roots of vegetables. There is an increasing trend of eating at fast food restaurants serving western style food like pizzas and burgers, as well as consumption of processed food like noodles and biscuits that are high in salt, sugar and fat. It has been proposed that Nepal has entered the fourth stage of "nutrition transition" ${ }^{6}$ which is the "degenerative diseases" pattern, as a result of which overweight, obesity and non communicable diseases is rapidly growing. As the NCD study indicates, 99.6\% of the population had at least one risk factor of non communicable disease and predicts that the burden will become "unbearable" if not addressed in time. The WHO Non-Communicable diseases country profiles 2018 shows $30 \%$ of total deaths in Nepal to be associated with cardiovascular disease and $13 \%$ with other $\mathrm{NCDs}^{7}$.

There seems to be growing awareness amongst the urban population to eat healthy, be more physically active and the consequences of obesity. More original studies correlating obesity with various pathological conditions in Nepal are needed. The country has to be aware that the BMI cut off for obesity related cardiovascular disease and T2DM as determined by World Health Organization $\left(>30 \mathrm{~kg} / \mathrm{m}^{2}\right)$ is in fact lower for Asian populations ${ }^{8}$. A majority of Asians with high risk of CVD and T2DM have a BMI between 22 and 25 $\mathrm{kg} / \mathrm{m}^{2}$. The best and most cost effective way to address this rising trend in obesity related diseases in Nepal is to revert back to the original, traditional Nepali food.

\section{REFERENCES}

1. Woods S. C., Seeley R. J., Porte D., Jr., Schwartz M. W. Signals that regulate food intake and energy homeostasis. PubMed - NCBI [Internet]. 1998[cited 2019 Jun 30]. 280(5368):1378-83. [PubMed]

2. Chaudhri O, Small C, Bloom S. Gastrointestinal hormones regulating appetite. Philos Trans $\mathrm{R}$ Soc Lond B Biol Sci. 2006 Jul 29;361(1471):1187-209. [PubMed]

3. Singla $P$, Bardoloi A, Parkash AA. Metabolic effects of obesity: A review. World J Diabetes. 2010 Jul 15;1(3):7688. [DOI]

4. Sam S, Mazzone T. Adipose tissue changes in obesity and the impact on metabolic function. Transl Res. 2014 Oct;164(4):284-92. [DOI]

5. World Health Organization: Noncommunicable diseases in the South-East Asia region: situation and response 2011. 2011. New Delhi: World Health Organization;2011. 104p.[Full Text]

6. Subedi YP, Marais D, Newlands D. Where is Nepal in the nutrition transition? Asia Pac J Clin Nutr. 2017 Mar;26(2):358-67. https://www.who.int/nmh/ countries/2018/npl_en.pdf [Full Text]

8. Appropriate body-mass index for Asian populations and its implications for policy and intervention strategies. - PubMed - NCBI [Internet]. [cited 2019 Jul 1]. Available from: https://www.ncbi.nlm.nih.gov/ pubmed/14726171[PubMed] 